\title{
Generalization of results about the Bohr radius for power series
}

\author{
by \\ Lev Aizenberg (Ramat-Gan)
}

\begin{abstract}
The Bohr radius for power series of holomorphic functions mapping Reinhardt domains $\mathcal{D} \subset \mathbb{C}^{n}$ into a convex domain $G \subset \mathbb{C}$ is independent of the domain $G$.
\end{abstract}

1. Preliminaries. Let us recall the theorem of H. Bohr [13] of 1914.

THEOREM 1.1. If a power series

$$
f\left(z_{1}\right)=\sum_{k=0}^{\infty} c_{k} z_{1}^{k}
$$

converges in the unit disk $U_{1}$ and its sum has modulus less than 1 , then

$$
\sum_{k=0}^{\infty}\left|c_{k} z_{1}^{k}\right|<1
$$

for $\left|z_{1}\right|<1 / 3$. Moreover, the constant $1 / 3$ cannot be improved.

For convenience we write the inequality (1.2) in the following equivalent form:

$$
\sum_{k=1}^{\infty}\left|c_{k} z_{1}^{k}\right|<1-\left|c_{0}\right| .
$$

Several generalizations of this result have been obtained.

$1^{\mathrm{o}}([24])$. If the sum of the series (1.1) is such that $\left|\Re f\left(z_{1}\right)\right|<1$ in $U_{1}$ and $c_{0}>0$, then the inequality (1.3) holds for $\left|z_{1}\right|<1 / 3$.

$2^{\circ}([23],[22])$. If $\Re f\left(z_{1}\right)<1$ in $U_{1}$ and $c_{0}>0$, then (1.3) holds for $\left|z_{1}\right|<1 / 3$.

$3^{\circ}([20])$. If $\Re\left\{[\exp (-i \arg f(0))] f\left(z_{1}\right)\right\}<1$ in $U_{1}$ (here we assume that $\arg f(0)=0$ if $f(0)=0)$ then (1.3) holds for $\left|z_{1}\right|<1 / 3$.

2000 Mathematics Subject Classification: Primary 32A05.

Key words and phrases: Bohr radius, Reinhardt domains, power series. 
Formulations of Bohr's theorem in several complex variables appeared very recently. We recall some of them.

Given a complete Reinhardt domain $\mathcal{D}$, we denote by $R_{1}(\mathcal{D})$ the largest nonnegative number $r$ with the property that if the power series

$$
f(z)=\sum_{|\alpha| \geq 0} c_{\alpha} z^{\alpha}, \quad z \in \mathcal{D},
$$

where $\alpha=\left(\alpha_{1}, \ldots, \alpha_{n}\right),|\alpha|=\alpha_{1}+\cdots+\alpha_{n}, z^{\alpha}=z_{1}^{\alpha_{1}} \ldots z_{n}^{\alpha_{n}}$ and all $\alpha_{i}$ are nonnegative integers, converges in $\mathcal{D}$ and the modulus of its sum is less than 1 , then

$$
\sum_{|\alpha| \geq 1}\left|c_{\alpha} z^{\alpha}\right|<1-\left|c_{0}\right|
$$

in the homothetic domain $\mathcal{D}_{r}=r \mathcal{D}$. Here $c_{0}=c_{0, \ldots, 0}$. If $\mathcal{D}$ is moreover bounded, we define $R_{2}(\mathcal{D})$ similarly, with the last inequality replaced by

$$
\sum_{|\alpha| \geq 1} \sup _{\mathcal{D}_{r}}\left|c_{\alpha} z^{\alpha}\right|<1-\left|c_{0}\right| .
$$

We call $R_{1}(\mathcal{D})$ (resp. $R_{2}(\mathcal{D})$ ) the first (resp. second) Bohr radius of $\mathcal{D}$.

Let

$$
\mathcal{D}_{p}^{n}=\left\{z \in \mathbb{C}^{n}:\left|z_{1}\right|^{p}+\cdots+\left|z_{n}\right|^{p}<1\right\}
$$

where $0<p \leq \infty$. The domain $\mathcal{D}_{\infty}^{n}$ is the poly-disk $\left\{z \in \mathbb{C}^{n}:\left|z_{j}\right|<1, j=\right.$ $1, \ldots, n\}$.

Theorem 1.2 ([12], see also [18]). For $n>1$ one has

$$
\frac{1}{3 \sqrt{n}}<R_{1}\left(\mathcal{D}_{\infty}^{n}\right)<\frac{2 \sqrt{\log n}}{\sqrt{n}} .
$$

Theorem $1.3([1])$. For $n>1$ one has

$$
\frac{1}{3 \sqrt[3]{e}}<R_{1}\left(\mathcal{D}_{1}^{n}\right) \leq \frac{1}{3}
$$

The estimates (1.5) and (1.6) were generalized to $R_{1}\left(\mathcal{D}_{p}^{n}\right)$ for $1 \leq p<\infty$ in [11] and for $0<p \leq 1$ in [3]. We point out the following new remarkable result which improves the lower estimate in (1.5).

Theorem $1.4([15])$. For $n>1$ one has

$$
C \sqrt{\frac{\log n}{n \log \log n}}<R_{1}\left(\mathcal{D}_{\infty}^{n}\right),
$$

where the constant $C$ is independent of $n$.

Both Bohr radii coincide for a polydisk, and in the case $n=1$ they coincide with the classical Bohr radius $1 / 3$. If $\mathcal{D}$ is not a polydisk, then naturally $R_{2}(\mathcal{D})<R_{1}(\mathcal{D})$. 
TheORem 1.5 ([1]). For every complete bounded Reinhardt domain $\mathcal{D}$,

$$
1-\sqrt[n]{2 / 3}<R_{2}(\mathcal{D})
$$

TheOREM $1.6([1])$. One has

$$
R_{2}\left(\mathcal{D}_{1}^{n}\right)<\frac{0.44663}{n} .
$$

The radius $R_{2}(\mathcal{D})$ was investigated in [11], [17]. Other results about the Bohr radius for holomorphic functions can be found in [2], [5], [7]-[10]. We would like to call a special attention to the paper [16] in which the relation between the Bohr radius and the Banach-Mazur distance between Banach spaces was discovered.

2. Generalized Bohr radii. One of the proofs of Bohr's theorem (Theorem 1.1) is based on the Landau inequality [21]: if the function (1.1) satisfies in $U_{1}$ the inequality $\left|f\left(z_{1}\right)\right|<1$, then $\left|c_{k}\right| \leq 2\left(1-\left|c_{0}\right|\right)$ for every $k \geq 1$. This inequality can be obtained as a simple consequence of the Carathéodory inequality [14]: if the function (1.1) satisfies in $U_{1}$ the inequality $\Re f\left(z_{1}\right)>0$, then $\left|c_{k}\right| \leq 2 \Re c_{0}$ for every $k \geq 1$. Both inequalities are particular cases of a more general assertion.

Let $\widetilde{G}$ be the convex hull of $G$.

Proposition 2.1 ([3]). If $f\left(U_{1}\right) \subset G$, then

$$
\left|c_{k}\right| \leq 2 \operatorname{dist}\left(c_{0}, \partial \widetilde{G}\right) \quad \text { for all } k \geq 1 \text {. }
$$

Now it is not difficult to prove a generalization of Theorem 1.1. Let $G \subset \mathbb{C}$ be any domain. A point $p \in \partial G$ is called a point of convexity if $p \in \partial \widetilde{G}$. A point of convexity $p$ is called regular if there exists a disk $U \subset G$ so that $p \in \partial U$.

THEOREM 2.1. If the function (1.1) is such that $f\left(U_{1}\right) \subset G$, with $\widetilde{G} \neq \mathbb{C}$, then

$$
\sum_{k=1}^{\infty}\left|c_{k} z_{1}^{k}\right|<\operatorname{dist}\left(c_{0}, \partial \widetilde{G}\right)
$$

for $\left|z_{1}\right|<1 / 3$. The constant $1 / 3$ cannot be improved if $\partial G$ contains at least one regular point of convexity.

Proof. 1) If $\left|z_{1}\right|<1 / 3$ then (2.1) yields

$$
\sum_{k=1}^{\infty}\left|c_{k} z_{1}^{k}\right|<2 \operatorname{dist}\left(c_{0}, \partial \widetilde{G}\right) \sum_{k=1}^{\infty} \frac{1}{3^{k}}=\operatorname{dist}\left(c_{0}, \partial \widetilde{G}\right) .
$$

2) We now prove the exactness of the constant $1 / 3$ in case the boundary contains at least one regular point of convexity. In the classical case 
of Bohr's Theorem 1.1 this is obtained by considering the family of functions $([21])$

$$
f\left(z_{1}\right)=\frac{\alpha-z_{1}}{1-\alpha z_{1}}, \quad 0<\alpha<1
$$

Here

$$
\sum_{k=1}^{\infty}\left|c_{k} z_{1}^{k}\right|=1 \quad \text { if and only if } \quad\left|z_{1}\right|=\frac{1}{1+2 \alpha} .
$$

Letting $\alpha \rightarrow 1$, we obtain the desired result. Note that instead of (2.3) one can use the family $e^{i \phi} f\left(z_{1}\right)$, where $f\left(z_{1}\right)$ is taken from (2.3). In this case $c_{0}=e^{i \phi} \alpha$, and when $\alpha \rightarrow 1$ we see that $c_{0}$ tends to $\partial U_{1}$ along the radius of argument $\phi$. If $G$ is an arbitrary disk $U$, then, remarking that (2.2) does not change under homotheties and translations, we deduce the exactness of $1 / 3$ for any disk. Let $\zeta$ be a regular point of convexity. Then there exists a disk $U \subset G$ such that $\zeta \in \partial U \cap \partial G$. Consider the functions $f$ in (1.1) such that $f\left(U_{1}\right) \subset U$. For suitable $c_{0}$ (see above) we have $\operatorname{dist}\left(c_{0}, \partial U\right)=\operatorname{dist}\left(c_{0}, \partial G\right)=\operatorname{dist}\left(c_{0}, \partial \widetilde{G}\right)$. Therefore, in the inequality (2.2) one cannot take $\left|z_{1}\right|<r$, where $r>1 / 3$.

We remark that Theorem 1.1, assertion $3^{\circ}$, as well as generalizations of $1^{\circ}$ and $2^{\circ}$ are contained in Theorem 2.1. For example, in $1^{\circ}$ there is no need to assume $c_{0}>0$, and instead of (1.3) one gets

$$
\sum_{k=1}^{\infty}\left|c_{k} z_{1}^{k}\right|<1-\left|\Re c_{0}\right| .
$$

Similarly in $2^{\circ}$ there is no need to assume $c_{0}>0$, and instead of (1.3) one gets

$$
\sum_{k=1}^{\infty}\left|c_{k} z_{1}^{k}\right|<1-\Re c_{0} .
$$

Let us recall another fact, known earlier:

$4^{\mathrm{o}}([4])$. If $\Re f\left(z_{1}\right)>0$ in $U_{1}$ and $c_{0}>0$, then one has

$$
\sum_{k=1}^{\infty}\left|c_{k} z_{1}^{k}\right|<c_{0}
$$

for $\left|z_{1}\right|<1 / 3$, and the constant $1 / 3$ cannot be improved.

I thought before that Theorem 1.1 and $4^{\circ}$ were two different facts involving the same Bohr radius. Now I know that both results are particular cases of Theorem 2.1. Namely, in the case of $4^{\circ}$, without the assumption $c_{0}>0$, we 
get

$$
\sum_{k=1}^{\infty}\left|c_{k} z_{1}^{k}\right|<\Re c_{0}
$$

instead of (2.4).

Theorem 2.1 motivates the following generalization of the first and second Bohr radii. Denote by $R_{1}(\mathcal{D}, G)$ (resp. $R_{2}(\mathcal{D}, G)$ ), where $G \subset \mathbb{C}, \widetilde{G} \neq \mathbb{C}$, and $\mathcal{D}$ is a complete Reinhardt domain (resp. a bounded complete Reinhardt domain) in $\mathbb{C}^{n}$, the largest $r \geq 0$ such that if the function (1.4) is holomorphic in $\mathcal{D}$ and $f(\mathcal{D}) \subset G$ then

$$
\sum_{|\alpha| \geq 1}^{\infty}\left|c_{\alpha} z^{\alpha}\right|<\operatorname{dist}\left(c_{0}, \partial \widetilde{G}\right)
$$

in a homothety $\mathcal{D}_{r}$ (respectively

$$
\left.\sum_{|\alpha| \geq 1}^{\infty} \sup _{\mathcal{D}_{r}}\left|c_{\alpha} z^{\alpha}\right|<\operatorname{dist}\left(c_{0}, \partial \widetilde{G}\right)\right) .
$$

Theorem 2.1 and the result from [6] about the Rogosinski radius allow one to hope that the two Bohr radii $R_{1}(\mathcal{D}, G)$ and $R_{2}(\mathcal{D}, G)$ are independent of the convex domain $G$. The main result of the present paper is the proof of this more general assertion.

3. The main result. Let $M$ be a complex manifold, and let $\mathcal{H}(M)$ be the space of functions holomorphic on $M$, equipped with the natural topology of uniform convergence on compact subsets of $M$.

Let $\|\cdot\|_{r}, r \in(0,1)$, be a one-parameter family of seminorms in $\mathcal{H}(M)$ that are continuous with respect to the topology of $\mathcal{H}(M)$. In what follows we always assume that

(a) $\|\cdot\|_{r_{1}} \leq\|\cdot\|_{r_{2}}$ if $r_{1} \leq r_{2}$.

(b) $\|f \cdot g\|_{r} \leq\|f\|_{r} \cdot\|g\|_{r}$ for all $r \in(0,1)$,

and there exists a point $z_{0} \in M$ such that

(c) $\|f\|_{r} \rightarrow\left|f\left(z_{0}\right)\right|$ as $r \rightarrow 0$, for all $f \in \mathcal{H}(M)$,

(d) $\|f\|_{r}=\left|f\left(z_{0}\right)\right|+\left\|f-f\left(z_{0}\right)\right\|_{r}$, for all $f \in \mathcal{H}(M)$.

Denote by $B\left(\left\{\|\cdot\|_{r}\right\}, G\right)$ the largest $r \geq 0$ such that for each $f \in \mathcal{H}(M)$ with $f(M) \subset G$ one has

$$
\left\|f-f\left(z_{0}\right)\right\|_{r}<\operatorname{dist}\left(f\left(z_{0}\right), \partial \widetilde{G}\right),
$$

where $\widetilde{G}$ is the convex hull of the domain $G \subset \mathbb{C}$. 
Proposition 3.1. If $U$ is any disk and $\Pi$ is any half-plane, then

$$
B\left(\left\{\|\cdot\|_{r}\right\}, \Pi\right)=B\left(\left\{\|\cdot\|_{r}\right\}, U\right) .
$$

Proof. Let $\Pi_{1}=\left\{z_{1}: \Re z_{1}>0\right\}$. Then $([4$, Theorem 7$])$

$$
B\left(\left\{\|\cdot\|_{r}\right\}, U_{1}\right)=B^{\prime}\left(\left\{\|\cdot\|_{r}\right\}, \Pi_{1}\right),
$$

where $B^{\prime}$ is defined in the same way as $B$ but with the additional assumption $f\left(z_{0}\right)>0$. This assumption can be removed as follows. If $\Re f\left(z_{0}\right)>0$ in $M$ then $\Re f_{1}\left(z_{0}\right)>0$, where $f_{1}(z)=f(z)-\Im f\left(z_{0}\right)$. But $f_{1}\left(z_{0}\right)>0$, hence

$$
B\left(\left\{\|\cdot\|_{r}\right\}, U_{1}\right)=B\left(\left\{\|\cdot\|_{r}\right\}, \Pi_{1}\right) .
$$

We remark that (3.1) does not change under homotheties, translations and rotations of the domain $G$. Therefore (3.2) holds.

Theorem 3.1. If $\widetilde{G} \neq \mathbb{C}$, then $B\left(\left\{\|\cdot\|_{r}\right\}, G\right)$ is not smaller than (3.2). If $\partial G$ contains at least one regular point of convexity, then $B\left(\left\{\|\cdot\|_{r}\right\}, G\right)$ is equal to (3.2).

Proof. Let $\widetilde{G} \neq \mathbb{C}$ and $f(M) \subset G$. Fix any $f\left(z_{0}\right) \in G$. On the boundary $\partial \widetilde{G}$ there exists a point $\zeta$ so that $\operatorname{dist}\left(f\left(z_{0}\right), \partial \widetilde{G}\right)=\operatorname{dist}\left(f\left(z_{0}\right), \zeta\right)$. Through the point $\zeta$ passes the line of support of $\widetilde{G}$ which defines a half-plane $\Pi_{0} \supseteq G$. Then

$$
\operatorname{dist}\left(f\left(z_{0}\right), \partial \widetilde{G}\right)=\operatorname{dist}\left(f\left(z_{0}\right), \partial \Pi_{0}\right) .
$$

Therefore $B\left(\left\{\|\cdot\|_{r}\right\}, G\right) \geq B\left(\left\{\|\cdot\|_{r}\right\}, \Pi_{0}\right)$, since $\{f \in \mathcal{H}(M): f(M) \subset G\} \subset$ $\left\{f \in \mathcal{H}(M): f(M) \subset \Pi_{0}\right\}$.

Assume now that there is a regular point of convexity in $\partial G$. Then the proof repeats part 2) of the proof of Theorem 2.1. Note that there we did not use the concrete form of the family (2.3), but rather the fact that $c_{0}$ can lie on any radius emanating from the center of the disk $U$ to its boundary. So, assume that $U \subset G$ and $\zeta \in \partial U \cap \partial G \cap \partial \widetilde{G}$. Consider $f\left(z_{0}\right)$ lying on the radius from the center of $U$ to $\zeta$. Then $\operatorname{dist}\left(f\left(z_{0}\right), \zeta\right)=$ $\operatorname{dist}\left(f\left(z_{0}\right), \partial U\right)=\operatorname{dist}\left(f\left(z_{0}\right), \partial \widetilde{G}\right)$, hence $B\left(\left\{\|\cdot\|_{r}\right\}, G\right) \leq B\left(\left\{\|\cdot\|_{r}\right\}, U\right)$, since $\{f \in \mathcal{H}(M): f(M) \subset U\} \subset\{f \in \mathcal{H}(M): f(M) \subset G\}$.

Corollary 3.1. If the domain $G$ is convex and $G \neq \mathbb{C}$, then $B\left(\left\{\|\cdot\|_{r}\right\}, G\right)$ is independent of the choice of $G$.

Proof. There exists a disk $U \subset G$ such that $\partial U \cap \partial G \neq \emptyset$. Therefore there exist regular points of convexity on $\partial G$.

Corollary 3.2. The first Bohr radius $R_{1}(\mathcal{D}, G)$ and the second Bohr radius $R_{2}(\mathcal{D}, G)$ are independent of the choice of the convex domain $G$, $G \neq \mathbb{C}$. 
In particular, the assertions of Theorems 1.2-1.4 are valid for $R_{1}(\mathcal{D}, G)$ while those of Theorems 1.5 and 1.6 are valid for $R_{2}(\mathcal{D}, G)$ for every convex domain $G \neq \mathbb{C}$.

Some concluding remarks. If the family of seminorms $\|\cdot\|_{r}$ does not satisfy some of the conditions (a)-(d), then the assertion of Theorem 3.1 is not valid anymore. Examples can be found in [4]. If $\widetilde{G}=\mathbb{C}$, then the right-hand side of (3.1) is equal to $\infty$, therefore in this case

$$
B\left(\left\{\|\cdot\|_{r}\right\}, G\right)=1 .
$$

One can also consider different realizations of $B\left(\left\{\|\cdot\|_{r}\right\}, G\right)$ than the first and second Bohr radii $R_{1}(\mathcal{D}, G)$ and $R_{2}(\mathcal{D}, G)$.

We conclude the present article with an open problem: if $\widetilde{G} \neq \mathbb{C}$, is it always true that $B\left(\left\{\|\cdot\|_{r}\right\}, G\right)$ is equal to (3.2)? The same question makes sense for the first and second Bohr radii $R_{1}(\mathcal{D}, G)$ and $R_{2}(\mathcal{D}, G)$.

Acknowledgements. The author is deeply grateful to E. Liflyand and A. Vidras for their help in preparing the paper and improving the presentation.

\section{References}

[1] L. Aizenberg, Multidimensional analogues of Bohr's theorem on power series, Proc. Amer. Math. Soc. 128 (2000), 1147-1155.

[2] —, Bohr Theorem, in: Encyclopedia of Mathematics, Supplement II, M. Hazewinkel (ed.), Kluwer, Dordrecht, 2000, 76-78.

[3] -, Generalization of Carathéodory's inequality and the Bohr radius for multidimensional power series, in: Oper. Theory Adv. Appl. 158, Birkhäuser, 2005, 87-94.

[4] L. Aizenberg, A. Aytuna and P. Djakov, An abstract approach to Bohr's phenomenon, Proc. Amer. Math. Soc. 128 (2000), 2611-2619.

[5] - - - - , Generalization of Bohr's theorem for bases in spaces of holomorphic functions of several complex variables, J. Math. Anal. Appl. 258 (2001), 428-447.

[6] L. Aizenberg, M. Elin and D. Shoikhet, On the Rogosinski radius for holomorphic mappings and some of its applications, Studia Math. 128 (2005), 147-158.

[7] L. Aizenberg, I. B. Grossman and Yu. F. Korobeĭnik, Some remarks on Bohr radius for power series, Izv. Vyssh. Ucheb. Zav. Mat. 2002, no. 10, 3-10 (in Russian).

[8] L. Aizenberg, E. Liflyand and A. Vidras, Multidimensional analogue of the van der Corput-Visser inequality and its application to the estimation of the Bohr radius, Ann. Polon. Math. 80 (2003), 47-54.

[9] L. Aizenberg and A. Vidras, On the Bohr radius of two classes of holomorphic functions, Siberian Math. J. 45 (2004), 606-617.

[10] C. Bénéteau, A. Dahlner and D. Khavinson, Remarks on the Bohr phenomenon, Comput. Methods Funct. Theory 4 (2004), 1-19.

[11] H. P. Boas, Majorant series, J. Korean Math. Soc. 37 (2000), 321-337.

[12] H. P. Boas and D. Khavinson, Bohr's power series theorem in several variables, Proc. Amer. Math. Soc. 125 (1997), 2975-2979. 
[13] H. Bohr, A theorem concerning power series, Proc. London Math. Soc. 13 (1914), $1-5$.

[14] C. Carathéodory, Über den Variabilitätsbereich der Koeffizienten von Potenzreihen, die gegebene Werte nicht annehmen, Math. Ann. 64 (1907), 95-115.

[15] A. Defant and L. Frerick, A logarithmic lower bound for multi-dimensional Bohr radii, Israel J. Math. 152 (2006), 17-28.

[16] A. Defant, D. Garcia and M. Maestre, Bohr's power series theorem and local Banach space theory, J. Reine Angew. Math. 557 (2003), 173-197.

[17] - - - - Estimates for the first and second Bohr radii of Reinhardt domains, J. Approx. Theory 128 (2004), 53-68.

[18] S. Dineen and R. Timoney, Absolute bases, tensor products, and a theorem of Bohr, Studia Math. 94 (1989), 227-234.

[19] P. B. Djakov and M. S. Ramanujan, A remark on Bohr's theorem and its generalizations, J. Anal. 8 (2000), 65-77.

[20] G. Kresin and V. Maz'ya, Sharp Bohr's type real part estimates, preprint, 2006, $16 \mathrm{pp}$.

[21] E. Landau und D. Gaier, Darstellung und Begründung einiger neuerer Ergebnisse der Funktionentheorie, Springer, 1986.

[22] V. I. Paulsen, G. Popescu and D. Singh, On Bohr inequality, Proc. London Math. Soc. 85 (2002), 493-515.

[23] S. Sidon, Über einen Satz von Herrn Bohr, Math. Z. 26 (1927), 731-732.

[24] M. Tomić, Sur un théorème de H. Bohr, Math. Scand. 11 (1963), 103-106.

Department of Mathematics

Bar-Ilan University

52900 Ramat-Gan, Israel

E-mail: aizenbrg@math.biu.ac.il

Received September 6, 2006

Revised version February 16, 2007 